\title{
Extension of near-wall domain decomposition to modeling flows with laminar-turbulent transition
}

DOI:

10.4208/CICP.OA-2021-0123

\section{Document Version}

Accepted author manuscript

Link to publication record in Manchester Research Explorer

\section{Citation for published version (APA):}

Petrov, M., Utyuzhnikov, S., Chikitkin, A. V., \& Smirnova, N. (2022). Extension of near-wall domain decomposition to modeling flows with laminar-turbulent transition. Communications in Computational Physics, 31(2), 645-668. https://doi.org/10.4208/CICP.OA-2021-0123

\section{Published in:}

Communications in Computational Physics

\section{Citing this paper}

Please note that where the full-text provided on Manchester Research Explorer is the Author Accepted Manuscript or Proof version this may differ from the final Published version. If citing, it is advised that you check and use the publisher's definitive version.

\section{General rights}

Copyright and moral rights for the publications made accessible in the Research Explorer are retained by the authors and/or other copyright owners and it is a condition of accessing publications that users recognise and abide by the legal requirements associated with these rights.

\section{Takedown policy}

If you believe that this document breaches copyright please refer to the University of Manchester's Takedown Procedures [http://man.ac.uk/04Y6Bo] or contact uml.scholarlycommunications@manchester.ac.uk providing relevant details, so we can investigate your claim.

\section{OPEN ACCESS}




\title{
Extension of near-wall domain decomposition to modeling flows with laminar-turbulent transition
}

\author{
M. Petrov ${ }^{1}$, S. Utyuzhnikov ${ }^{1,2}$, A. Chikitkin ${ }^{1}$ and N. Smirnova ${ }^{1}$ \\ 1 Moscow Institute of Physics \& Technology, Dolgoprudny 141700, Russia \\ 2 Department of Mechanical, Aerospace \& Civil Engineering, \\ the University of Manchester, Manchester, M13 9PL, U.K.,
}

\begin{abstract}
The near-wall domain decomposition method (NDD) has proved to be very efficient for modeling near-wall fully turbulent flows. In this paper the NDD is extended to non-equilibrium regimes with laminar-turbulent transition (LTT) for the first time. The LTT is identified with the use of the $e^{N}$-method which is applied to both incompressible and compressible flows. The NDD is modified to take into account LTT in an efficient way. In addition, implementation of the intermittency expands the capabilities of NDD to model non-equilibrium turbulent flows with transition. Performance of the modified NDD approach is demonstrated on various test problems of subsonic and supersonic flows past a flat plate, a supersonic flow over a compression corner and a planar shock wave impinging on a turbulent boundary layer. The results of modeling with and without decomposition are compared in terms of wall friction and show good agreement with each other while NDD significantly reducing computational resources needed. It turns out that the NDD can reduce the computational time as much as three times while retaining practically the same accuracy of prediction.
\end{abstract}

Key words: Domain decomposition; laminar-turbulent transition; interface boundary condition; near-wall flow; low-Reynolds-number model

\section{Introduction}

The problem of modeling a laminar-turbulent transition (LTT) is of great interest in the aircraft design since the turbulence of flow can have a significant impact on aerodynamic characteristics. In industrial applications, the direct numerical simulation (DNS) and large eddy simulation (LES) methods are not often used for modeling LTT. Instead, models based on the Reynolds-Averaged Navier-Stokes equations (RANS) are applied. In such models, as a rule, the LTT is set explicitly. LTT can be instant as in the Spalart-Allmaras one-equation model with the trip term [1]. On the other hand, some other models can take into account a transition region between the laminar and turbulent parts of the flow [2]. Regardless of a RANS model, computations can be time-consuming. At the same time, 
calculations in the near-wall region can take up to $90 \%$ of all time expenses. This is caused by the requirement to resolve a very thin laminar sublayer which is always present thanks to the no-slip boundary condition and damping effect of the wall.

The classical way to reduce the computational time for RANS calculations is to use the wall functions. The use of wall functions allows us to avoid detailed resolution of the near-wall region and reduce the time cost. This approach represents a simplest way for domain decomposition when Dirichlet boundary conditions are prescribed at the cell center of the nearest to the wall cell in one way or another way. The wall functions are semiempirical and replace the effect of the wall for the outer flow. As well known, despite a certain success with this approach, the wall functions have a number of limitations. They inevitably contain parameters to be tuned. Most of them do not take into account the effect of pressure gradient and other forces, and the obtained solution can be significantly mesh sensitive. For these reasons, the applications of wall functions to flows with complex geometries and boundary layer separation are very problematic. There are some advanced wall functions (see e.g. [3-6]) in which some of these problems are resolved. However, they are not able to overcome all the problems since they have a very limited basis. In addition, the use of wall functions can be problematic in cases with LTT, since they presume the presence of a fully developed turbulence.

The near-wall domain decomposition (NDD) method [7-9,11] represents an alternative way to reduce the computational time. In this approach the computational domain is split into two blocks. One of these two blocks covers a near-wall region. Such kind of domain decomposition immediately follows from the physics of the problem when the inner region is characterised by both high-gradients of the solution and small scale in the normal to the wall direction. The solution of the entire problem is realized via a non-overlapping domain decomposition. The boundary-value problems in each region are linked with each other via interface boundary conditions (IBCs) of Robin type. In the original formulation of NDD, an approximate NDD (ANDD) is realized. This is achieved with the use of the Thin Boundary Layer Equations (TBLE) in the inner region. The IBCs are obtained via transfer of the boundary conditions from the wall to the interface boundary [39,40]. As can be shown, in one-dimensional case the Dirichlet boundary condition can be exactly transferred to the interface boundary in the form of an IBC of Robin type. The location of the interface boundary $y^{*}$ allows the trade-off between the accuracy and computational time to be efficiently controlled. As shown in [10] for incompressible flow in a diffuser, an abatement in accuracy as much as a few percents allows the computational time to be reduced by one order of magnitude. Recent investigations have shown the effectiveness of the ANDD method for both steady and unsteady compressible flows around complex geometries with separated flows and shock waves [12-15]. In [13] it was shown that the ANDD method converges almost three times faster than the approach in one block without decomposition. In addition, in [13] the exact near-wall domain decomposition (ENDD) was proposed. In this approach the full RANS equations are solved in both blocks, which makes it possible to reach the accuracy comparable to a single-block solution. However, the ENDD method yields to ANDD in terms of the computational cost. 
In all the papers cited above the turbulent boundary layer was supposed to be fully developed. So far, the NDD was successfully used to model LTT only in the framework of the boundary layer model [16]. An immediate application of NDD to modeling nonequilibrium flows was problematic because of high dependence of IBC on the turbulent viscosity coefficient. In the current paper, the NDD is extended to the full computational region including the outer region (with respect to the boundary layer) with a predominant convection. The NDD is applied to the compressible Navier-Stokes equations in such a way that the LTT is identified by $e^{N}$ method and non-equilibrium turbulent flow is then simulated with the use of RANS.

The paper is organized as follows. In Section 2 the governing equations are formulated. The numerical method used for solving the unsteady nonlinear equations is described in Section 3. In Section 4 the use of NDD in the form of ANDD and ENDD for modeling LTT is addressed. In Section 5 the method for predicting LTT is presented. Section 6 contains computational tests such as sub- and supersonic flow past a flat plate, a supersonic flow over compression corner and a planar shock wave impinging on a turbulent boundary layer. The results obtained with the use of NDD are compared with the single-block solutions obtained without decomposition. Finally, conclusions are given in Section 7.

\section{Governing equations}

Gas state at space point $x=\left(x_{1}, x_{2}, x_{3}\right)^{T}$ and time moment $t$ is characterized by density $\rho$, velocity $\boldsymbol{u}=\left(u_{1}, u_{2}, u_{3}\right)^{T}$, pressure $p$ and energy per unit volume $E$.

In the dimensional form the governing equations are given by

$$
\begin{gathered}
\frac{\partial}{\partial t} \boldsymbol{U}+\nabla\left(\boldsymbol{F}-\boldsymbol{F}^{v}\right)=\boldsymbol{S}(\boldsymbol{U}), \\
\boldsymbol{F}=\left(\boldsymbol{F}_{1}, \boldsymbol{F}_{2}, \boldsymbol{F}_{3}\right), \quad \boldsymbol{F}^{v}=\left(\boldsymbol{F}_{1}^{v}, \boldsymbol{F}_{2}^{v}, \boldsymbol{F}_{3}^{v}\right),
\end{gathered}
$$

where $\boldsymbol{U}$ is the vector of conserved variables, $\boldsymbol{F}, \boldsymbol{F}^{v}$ are tensors of convective and viscous fluxes, $S$ is the source term:

$$
\begin{gathered}
\boldsymbol{U}=\left(\begin{array}{c}
\rho \\
\rho u_{1} \\
\rho u_{2} \\
\rho u_{3} \\
E \\
\rho \tilde{v}
\end{array}\right), \quad \boldsymbol{F}_{k}=\left(\begin{array}{c}
\rho u_{k} \\
\rho u_{1} u_{k}+\delta_{1 k} p \\
\rho u_{2} u_{k}+\delta_{2 k} p \\
\rho u_{3} u_{k}+\delta_{3 k} p \\
(E+p) u_{k} \\
\rho \tilde{v} u_{k}
\end{array}\right), \\
\boldsymbol{F}_{k}^{v}=\left(\begin{array}{c}
0 \\
0 \\
\tau_{1 k} \\
\tau_{2 k} \\
\tau_{3 k} \\
u_{\alpha} \tau_{\alpha k}-q_{k} \\
F_{k}^{v}(6)
\end{array}\right), \quad S=\left(\begin{array}{c} 
\\
0 \\
0 \\
S_{s a}
\end{array}\right) .
\end{gathered}
$$


Here, $\tau_{i j}, q_{k}$ are components of stress tensor and heat flux vector; $p=\rho R_{g} T$ ( $R_{g}$ is the gas constant); $E=\rho\left(e+\frac{1}{2} u_{\alpha} u_{\alpha}\right)$, where $e$ is the internal energy per unit mass; a summation is supposed over repeating indexes (Einstein rule); $\tilde{v}$ is the turbulent variable; $F_{k}^{v}(6)=$ $\frac{\rho}{\sigma}\left((v+\tilde{v}) \frac{\partial \tilde{v}}{\partial x_{k}}\right) ; S_{s a}$ is a turbulent source term.

For modeling turbulent flows the Spalart-Allmaras one-equation model with the trip term (SA-la) is used [1]. The full viscosity is given by $\mu=\mu_{m o l}+\mu_{t}$; the full heat conductivity, $\lambda=\lambda_{m o l}+\lambda_{t}$, where $\mu_{m o l}$ is the molecular viscosity; $\mu_{t}$ is the turbulent viscosity; $\lambda_{\text {mol }}$, the molecular heat conductivity; $\lambda_{t}$, the turbulent heat conductivity.

The turbulent viscosity is defined as

$$
\mu_{t}=\rho \tilde{v} f_{v 1}
$$

In turn, the turbulent source term reads:

$$
\begin{aligned}
& S_{s a}=c_{b 1}\left(1-f_{t 2}\right) \rho \tilde{S} \tilde{v}+\left(c_{w 1} f_{w}-\frac{c_{b 1}}{\kappa^{2}} f_{t 2}\right) \times \\
& \rho\left(\frac{\tilde{v}}{d}\right)^{2}+c_{b 2} \frac{\rho}{\sigma} \frac{\partial \tilde{v}}{\partial x_{k}} \frac{\partial \tilde{v}}{\partial x_{k}}+f_{t 1} \Delta U^{2} .
\end{aligned}
$$

Here, the last term $f_{t 1} \Delta U^{2}$ is used to model LTT where

$$
\begin{aligned}
& f_{t 1}=c_{t 1} g_{t} \exp \left(-c_{t 2} \frac{\omega_{t}^{2}}{\Delta U}\left(d^{2}+g_{t}^{2} d_{t}^{2}\right)\right), \\
& g_{t}=\min \left[0.1, \frac{\Delta U}{\omega_{t} \Delta x_{t}}\right], \quad c_{t 1}=1, \quad c_{t 2}=2 .
\end{aligned}
$$

In addition, $\Delta U$ is the difference between the velocity at the field point and that at the trip (on the wall); $\Delta x_{t}$, the grid spacing along the wall at the trip; $\omega_{t}$, the wall vorticity at the trip; $d_{t}$, the distance from the field point to the trip.

The other terms in the turbulent source are the following: 


$$
\begin{aligned}
& \tilde{S}=S+\frac{\tilde{v}}{\kappa^{2} d^{2}} f_{v 2}, \\
& S=\sqrt{2 W_{i j} W_{i j}}, W_{i j}=\frac{1}{2}\left(\frac{\partial u_{i}}{\partial x_{j}}-\frac{\partial u_{j}}{\partial x_{i}}\right), \\
& f_{v 1}=\frac{\chi^{3}}{\chi^{3}+c_{v 1}^{3}}, \quad \chi=\frac{\tilde{v}}{v_{m o l}}, \\
& f_{v 2}=1-\frac{\chi}{1+\chi f_{v 1}}, \\
& f_{t 2}=c_{t 3} \exp \left(c_{t 4} \chi^{2}\right), \\
& f_{w}=g\left[\frac{1+c_{w 33}^{6}}{g^{6}+c_{w 3}^{6}}\right]^{1 / 6}, \\
& g=r+c_{w 2}\left(r^{6}-r\right), r=\min \left(\frac{\tilde{v}}{\tilde{S} \kappa^{2} d^{2}}, 10\right),
\end{aligned}
$$

where

$$
\begin{array}{ccc}
c_{b 1}=0.1355 & c_{b 2}=0.622 & c_{v 1}=7.1 \\
c_{w 2}=0.3 & c_{w 3}=2 & c_{w 1}=\frac{c_{b 1}}{k^{2}}+\frac{1+c_{b 1}}{\sigma} \\
\sigma=2 / 3 & \kappa=0.41 & c_{t 3}=1.2 \\
c_{t 4}=0.5 & &
\end{array}
$$

Boundary conditions at the wall and free stream are of Dirichlet type and homogeneous.

The turbulent heat conduction coefficient is given by $\lambda_{t}=c_{p} \mu_{t} / \mathrm{Pr}_{t}$ with turbulent Prandtl number $\operatorname{Pr}_{t}=0.85$.

\section{Numerical method}

To solve system (2.1), an implicit finite-volume numerical method is used. It is implemented in the in-house code "FlowModellium" [17]. In the code, both structured and unstructured meshes can be used. Mesh cells can be tetrahedral, pyramidal, hexahedral, and prismatic.

Rewrite system by integrating it in each spatial cell $C_{i}$ in the computational domain and using the divergence theorem:

$$
\begin{aligned}
& \left(\bar{U}_{i}\right)_{t}=R_{i}=-\frac{1}{\left|C_{i}\right|} \sum_{f_{j} \in \mathcal{F}\left(C_{i}\right)}\left(\Phi_{j i}-\Phi_{j i}^{v}\right)+\bar{S}_{i}, \\
& i=1, \ldots, N_{c}
\end{aligned}
$$

where $\bar{U}_{i}$ is the cell average of the unknown vector. Inviscid fluxes $\Phi_{j i}$ are approximated using the Riemann problem solution. To solve the Riemann problem, a hybrid HLLHLLC $[18,19]$ solver is used. HLL is used on all mesh faces that are further from the body surface than a given distance. Viscous fluxes $\Phi_{j i}^{v}$ and the source term $\bar{S}_{i}$ are computed by 
using the approximation of involved spatial derivatives. Finally, system (3.1) becomes the system of nonlinear ODEs:

$$
\frac{d W(t)}{d t}=R(W(t))
$$

where

$$
W=\left[\begin{array}{c}
\bar{U}_{1} \\
\bar{U}_{2} \\
\ldots \\
\bar{U}_{N_{c}}
\end{array}\right], \quad R(W)=\left[\begin{array}{c}
R_{1}(W) \\
R_{2}(W) \\
\ldots \\
R_{N_{c}}(W)
\end{array}\right]
$$

The use of an implicit Euler approximation in time results in the following algebraic system of equations for time increments $\Delta W$

$$
\left[I-\Delta t \frac{\partial R}{\partial W}\left(W^{n}\right)\right] \Delta W \equiv A \Delta W=\Delta t R\left(W^{n}\right),
$$

which is solved using the LU-SGS scheme $[20,21]$. Its modifications in the context of the present method are discussed in [22,23]. The stationary solution is found by integrating over time until the following condition is satisfied:

$$
\|R\|<\epsilon,
$$

where $\epsilon$ is some small predefined parameter.

To solve the entire system of discrete governing equations, the NDD method is used in both variants: ANDD and ENDD. Formally, in both approaches the IBC are of Robin type. In addition, the equations in the outer block coincide. These two approaches are distinguished by the governing equations in the inner region. This is addressed in the next section.

\section{Near-wall domain decomposition}

In this section we consider sequentially basic ideas for implementation of both ANDD and ENDD. In addition, we discuss how to modify these methods for the LTT modeling.

First, consider TBLE used in ANDD. Each of these equations (apart from the continuity equation) can be written down as follows:

$$
\frac{\partial}{\partial y}\left(\mu \frac{\partial U}{\partial y}\right)=f
$$

Consider domain $0 \leq y \leq y_{e}$, and presume that equation (4.1) is completed by Dirichlet boundary conditions:

$$
U(0)=U_{0}
$$




$$
U\left(y_{e}\right)=U_{1}
$$

Let us split the entire region into the near-wall inner region $0 \leq y \leq y^{*}$ and outer region $y^{*} \leq y \leq y_{e}$. One can transfer the boundary condition from the wall at $y=0$ to the interface boundary $y=y^{*}[41]$ :

$$
U^{*}=\left.\frac{\partial U}{\partial y}\right|_{y^{*}} f_{1}+f_{2}+U_{0}
$$

where

$$
\begin{gathered}
f_{1}=\int_{0}^{y^{*}} \frac{\mu^{*}}{\mu} d y \\
f_{2}=-\int_{0}^{y^{*}} \frac{1}{\mu} \int_{0}^{y^{\prime}} f d y d y^{\prime}
\end{gathered}
$$

and $\mu^{*}=\mu\left(y^{*}\right)$.

Thus, the IBCs can be formulated in a universal form (4.3) for all variables but the normal velocity and density. For example, the IBC for the tangential velocity is obtained as [9]:

$$
v_{\tau}^{*}=f_{1}^{v} v_{\tau, y}^{*}+f_{2}^{v}
$$

which can be approximated as

$$
v_{\tau}^{*}=\frac{v_{\tau, I} f_{1}^{v}+d f_{2}^{v}}{d+f_{1}^{v}}
$$

Here, $v_{\tau}^{*}$ is the tangential velocity at the interface boundary; $v_{\tau, I}$, in the first cell near the interface in the outer region; $f_{1}^{v}$ and $f_{2}^{v}$, the integrals for the tangential component of velocity; $d$, the distance from the center of the first cell to the interface boundary. Once the solution in the outer block is computed, the profile of the tangential velocity in the inner domain can be obtained from the momentum conservation law with closure (4.4):

$$
v_{\tau}(y)=\left.\frac{d v}{d y}\right|_{y^{*}} \int_{0}^{y} \frac{\mu^{*}}{\mu(\xi)} d \xi-\int_{0}^{y} \frac{\frac{d p}{d x}\left(y^{*}-\xi\right)}{\mu(\xi)} d \xi
$$

The IBC for the normal component of velocity can be obtained from the Taylor expansion [13]:

$$
v_{n}=\frac{y^{*}}{3} v_{n}^{\prime}
$$

After approximating Eq. (4.6) near the interface boundary, the normal component of velocity near $y^{*}$ can be computed as

$$
v_{n}=\frac{y^{*}}{3 d+y^{*}} v_{n, I}
$$


where $v_{n, I}$ is the normal velocity at the interface with the outer region. Once the solution in the outer block is computed, the profile of the normal velocity can be obtained from

$$
v_{n}(y)=v_{n}\left(y^{*}\right)\left(\frac{y}{y^{*}}\right)^{2} .
$$

For the temperature the IBC reads:

$$
T^{*}=\frac{d T}{d y}\left(y^{*}\right) f_{1}^{T}+T_{w},
$$

where $f_{1}^{T}$ is the integral assigned to the temperature; $T^{*}$, the temperature at the interface; $T_{w}$, the surface temperature. Once the solution in the inner block is found, the temperature is computed as:

$$
T(y)=\left.\frac{d T}{d y}\right|_{y^{*}} \int_{0}^{y} \frac{k^{*}}{k(\xi)} d \xi+T_{w} .
$$

The density profile in the inner block is computed under the assumption of the constant pressure along the normal direction to the wall:

$$
\rho(y)=\frac{p_{I}}{T(y) R} .
$$

$p_{I}$ is the pressure in the first cell nearby the interface.

The viscosity profile in the inner block is used to compute integrals $f_{1}$ and $f_{2}$ in the boundary conditions of Robin type. In the ANDD method it is assumed that the profiles of turbulent viscosity and turbulent variable are known a priori. One of the profiles which can be used is the profile of Duprat et al. [24]. It takes into account the pressure gradient, which allows the separation point to be predicted more accurately. As an alternative profile, the profile from ZPG SA solution [25] can be used:

$$
\tilde{v}=\kappa u_{\tau} y .
$$

To model LTT with the SA-la model and ANDD, there are two options. At the first option it is assumed that the transition region is short. This assumption corresponds to the standard SA-la model. If such, the turbulent viscosity profile is set to zero in the inner region up to the transition onset (trip). Beyond that point the turbulent viscosity profile is supposed to correspond to a developed turbulence. The tests presented in Section 6 demonstrate that such an assumption is also applicable in the transition region, and it slightly distorts the solution. The results corresponding to this case are denoted by ANDDS. In the second option the transition region is determined by the intermittency coefficient.

The intermittency is expressed as

$$
\gamma_{I}(x)=1-0.01^{\left(x-x_{1}\right)^{2} /\left(x_{2}-x_{1}\right)^{2}} .
$$


Here $x_{1}$ is the transition onset, $x_{2}$ is the transition end. In [26] it is shown that (4.11) is a reasonably good approximation for the intermittency coefficient.

In this case, within the transition region between $x_{1}$ and $x_{2}$, the turbulent viscosity profile is multiplied by $\gamma_{I}$. It is set to zero in the inner region up to the transition onset. After the transition end the turbulent viscosity profile corresponds to a developed turbulence. This approach allows the transition region to be taken into account, and thereby it expands the capabilities of the SA-la model to modeling flows with extended transition regions. In Section 6 the appropriate results are presented by ANDD-I.

To compute the turbulent viscosity profile, the surface friction is needed. It can be computed by integrating the momentum equation in the near-wall region straightforward:

$$
\tau_{w}=\mu \frac{\partial v_{\tau}}{\partial y}=\frac{\mu^{*}}{f_{1}^{v}}\left(v_{\tau}^{*}-f_{2}^{v}\right)-\frac{d p}{d x} y^{*} .
$$

As a result, the general framework for solving the problem with the ANDD method can be formulated as follows:

1. specify the initial flow field in both inner and outer blocks;

2. in the inner block the Thin Boundary Layer (TBL) model is used to obtain the boundary condition of Robin type at the interface boundary;

3. the problem is solved in the outer region using Robin boundary condition (4.3) at the interface boundary. This step corresponds to one global LU-SGS iteration of Eq. $(3.4)$;

4. once the solution in the outer region is found, the flow field in the inner region is recalculated with the Dirichlet boundary conditions obtained from the solution in the outer region;

5. if the convergence condition (3.5) in the outer region is not satisfied, return to step 2 .

The ENDD can be implemented as an addition to the ANDD. Now consider the equations solved in the ENDD. They entirely correspond to the full RANS equations in both the inner and outer regions. Let $u_{1}$ be the solution of the problem in the inner domain, $u_{2}$ be the solution in the outer domain. The solution of the single block problem is reduced to the iterative solution of two systems in the inner and outer regions:

$$
L\left(u_{1}\right)=R_{1}, \quad \frac{\partial u_{1}^{k+1}}{\partial n}+\sigma u_{1}^{k+1}=g_{1}^{k+1} \text { at } y=y^{*}
$$

and

$$
L\left(u_{2}\right)=R_{2}, \quad \frac{\partial u_{2}^{k+1}}{\partial n}-\sigma u_{2}^{k+1}=g_{2}^{k+1} \text { at } y=y^{*}
$$


where

$$
g_{1}^{k+1}=g_{2}^{k+1}+2 \sigma u_{2}^{k+1}, \quad g_{2}^{k+1}=g_{1}^{k}-2 \sigma u_{1}^{k}
$$

and

$$
g_{1}^{0}=\frac{\partial u_{1}^{0}}{\partial n}+2 \sigma u_{1}^{0}
$$

For each problem the IBCs are precisely satisfied for the exact solution. In the general form, they were introduced for the non-overlapping domain decomposition by Lions and Deng [27], [28]. The convergence of iteration process (4.12)-(4.14) for an arbitrary positive $\sigma$ in application to the Poisson equation was proven in [29]. To make these interface boundary conditions consistent with the IBC in ANDD, we select $\sigma=\frac{1}{f_{1}}$, where $f_{1}$ is the integral from the Robin boundary condition (4.3). This choice of $\sigma$ is based on the locally one-dimensional approximation of the Steklov-Poincaré operator [30].

One should note, in case the ENDD is used, the LTT modeling is provided directly by the turbulence model used. Conversely, in the ANDD the choice of a turbulent profile in the inner block depends on the part of the flow set. This is possible thanks to the fact that the full equations of the model are solved in both the inner and outer regions. Therefore, the formulation and implementation of the ENDD in the cases of developed turbulence and nonequilibrium flow with LTT are mostly identical.

\section{$5 e^{N}$-method to define transition from laminar to turbulent flow}

To model LTT using the SA-la model, the position of the LTT on the body surface is needed. To find this position, the $e^{N}$-method is used [31,32]. Consider now the main idea of the method. Accordingly to the linear stability analysis, we linearize the Navier-Stokes equations with respect to small perturbations. For this purpose, the primitive variables included in equations (2.1) are represented as follows

$$
\Phi(\mathbf{x}, t)=\bar{\Phi}(\mathbf{x})+\Phi^{\prime}(\mathbf{x}, t),
$$

where $\bar{\Phi}(\mathbf{x})$ is the unperturbed vector of the primitive variables, $\Phi=\left(u_{1}, u_{2}, p\right)^{T}$ in the incompressible case and $\Phi=\left(u_{1}, u_{2}, p, T, u_{3}\right)^{T}$ in the compressible case, $\Phi^{\prime}(\mathbf{x}, t)$ is an appropriate unsteady perturbation.

After linearization with respect to a locally parallel to the wall solution, small perturbations are considered in the form of a plane wave:

$$
\Phi^{\prime}(\mathbf{x}, t)=\hat{\Phi}(y) \exp i(\alpha x+\beta z-\omega t),
$$

where $\hat{\Phi}$ is an amplitude function; $\alpha$ and $\beta$ are the wavenumbers; $\omega$ is the cyclic frequency.

After substituting such a perturbation into the linearized Navier-Stokes equations, the linear system of partial differential equations is transformed to the system of ordinary differential equations:

$$
\left(A D^{2}+B D+C\right) \hat{\Phi}=0
$$


which is completed by homogeneous boundary conditions. Here, $D$ is the differentiation operator in the normal to the wall direction. In the incompressible case matrices $A, B$ and $C$ have dimension $3 \times 3$. In turn, in the compressible case they have dimension $5 \times 5$. In the software implementation, those matrices used for the incompressible case are built according to paper [33], while matrices for the compressible case are taken from [32]. All these matrices are provided in the Appendix.

Further, the boundary value problem with homogeneous boundary conditions (5.1), which determines the eigenvalue problem, is approximated by finite differences and solved in the spatial form. In the incompressible case a regular mesh is used. In the compressible case an irregular mesh is specified with a refinement to the plate, which is constructed in such a way that approximately $50 \%$ nodes are situated inside the boundary layer. Then, this mesh is displayed on a regular one with transform $y=\eta L /(g-\eta)$. Here, $\eta$ is a new variable, with respect to which the computational mesh is regular; $L, g$ are automatically selected constant values. In both cases the approximation on a regular mesh is conducted in the following manner:

$$
\begin{array}{r}
D \hat{\Phi}=\frac{\hat{\Phi}_{D}^{(i+1)}-\hat{\Phi}_{D}^{(i-1)}}{2 \Delta \eta}+O\left(\Delta \eta^{2}\right), \\
D^{2} \hat{\Phi}=\frac{\hat{\Phi}_{D}^{(i+1)}-2 \hat{\Phi}_{D}^{(i)}+\hat{\Phi}_{D}^{(i-1)}}{\Delta \eta^{2}}+O\left(\Delta \eta^{2}\right) .
\end{array}
$$

Here, $i$ is the index of a mesh node, subscript $D$ means an approximate value.

After substitution (5.2) into (5.1) an eigenvalue problem is considered with the complex wavenumber $\alpha$ as an eigenvalue while $\beta$ and $\omega$ are treated as real parameters. In the study, the solution to the eigenvalue problem was performed using the software package ARPACK [34]. The wavenumbers $\alpha$, which are found for unstable modes, are used then to calculate the amplification curves. In agreement with the $e^{N}$-method, LTT starts when the envelope of amplification curves crosses the threshold of $N$ that is a factor defined for a given flow. For a wide range of two-dimensional boundary layers the threshold can be expressed by Mack's interpolation formula [35]:

$$
N=2.13-6.18 \lg (\mathrm{Tu}),
$$

where $T u$ is the degree of turbulence with respect to the inflow velocity as a percentage. In computations carried out with the SA-la model, the transition onset found in this way is used as the trip.

In a similar way the transition end can be found but another threshold should be used:

$$
N=5-6.18 \lg (T u) .
$$

It is to be noted that the analytical approximations (5.3) and (5.4) were used for the sake of simplicity. In practice, such curves might be available. If not, they need to be obtained from experiments for similar cases.

Next, we consider some examples of searching for a transition onset in sub- and supersonic flows past a flat plate. 
5.1 Search of a transition in a subsonic flow past a flat plate.

A subsonic flow past a flat plate of length $L=1.5 \mathrm{~m}$ is considered. The parameters of the inflow are set as for air under normal conditions: pressure is $p_{\infty}=101325 \mathrm{~Pa}$, density is $\rho_{\infty}=1.214 \mathrm{~kg} / \mathrm{m}^{3}$ and the sound speed is $c=341.8 \mathrm{~m} / \mathrm{s}$. The velocity of inflow is $U_{\infty}=50$ $\mathrm{m} / \mathrm{s}$. First, a laminar calculation was carried out. Then, 24 points were selected along the entire length of the plate with a refinement towards the edge to obtain one-dimensional data sets along the normal to the plate at these selected points. The layer thickness of the one-dimensional set was 15 boundary layer thicknesses for each point. In each onedimensional set, a uniform mesh of 2000 nodes was specified. The study of grid convergence shows that this number of nodes is sufficient.

Fig. 1 demonstrates the dependence of the transition onset on the number of nodes. The calculations for the search for unstable modes were carried out for 20 frequencies in the range $f=\omega U_{\infty} /(2 \pi L)=20-160 \mathrm{KHz}$. In the two-dimensional incompressible case, it was assumed that $\beta=0$. In turn, Fig. 2 shows the amplification curves for considered frequencies. Accordingly to formula (5.3), the threshold $N$-factor for a given degree of turbulence $T u=0.12 \%$ is equal to $N=7.82$ and crosses the envelope of amplification curves at $x_{1}=0.785$ that corresponds to the transition onset. At the transition end, $N$ and $x_{2}$ are equal to 10.7 and 1.25, respectively. The obtained transition points are in a good agreement with the results of physical experiments [36].

\subsection{Search of a transition in a supersonic flow past a flat plate.}

Next, a supersonic flow past a flat plate of length $L=0.5 \mathrm{~m}$ and Mach number $M_{\infty}=1.47$ is considered. The parameters of the inflow are set as follows: the pressure is $p_{\infty}=19650$ $\mathrm{Pa}$, the density is $\rho_{\infty}=0.3373 \mathrm{~kg} / \mathrm{m}^{3}$, and the velocity of inflow is $U_{\infty}=419.814 \mathrm{~m} / \mathrm{s}$. First, a laminar flow was calculated. Then, similar to the subsonic case, 24 points were selected along the entire length of the plate with a refinement towards the edge to obtain one-dimensional data sets along the normal to the plate. The layer thickness of the onedimensional set was 40 boundary layer thicknesses for each point. In each one-dimensional set, an irregular mesh of 800 nodes was specified with a refinement to the plate. The computations for the search for unstable modes were carried out for 18 frequencies in the range of $f=3.9-23 \mathrm{MHz}$. For each frequency the wavenumber $\beta$ was chosen in such a way that the disturbance at this frequency was maximal. To find an appropriate wavenumber, a series of calculations was carried out in a given range of $\beta$. It turned out that the maximum was achieved with $\beta \approx 0.1 / \delta$, where $\delta$ is the boundary layer thickness. Fig. 3 represents the amplification curves for considered frequencies. Accordingly to the experiment [31] the threshold $N$-factor for onset is $N=3.15$. It crosses the envelope of amplification curves at $x_{1}=0.135$. In this case, at the transition end $N=5.12$ and $x_{2}=0.33$. Similar to the subsonic case, the predicted transition points are in a good agreement with the results of the physical experiments provided in [31]. 


\section{Test cases}

To model the LTT, the Spalart-Allmaras one-equation model with the trip term (SAla) is used. Four test problems are considered: a sub- and supersonic flow past a flat plate, a supersonic flow over compression corner and a planar shock wave impinging on a turbulent boundary layer. For each problem a single-block solution without decomposition is also obtained for the comparison purposes. In the calculations the first cell height in the boundary layer is selected such that $y^{+} \approx 1$. The two-block calculations are carried out with the exact NDD, approximate NDD with a short transition region (ANDD-S) and approximate NDD with the intermittency (ANDD-I). In the case of ANDD, in the inner region before the transition a laminar viscosity profile is set. Finally, in the two-block solution the distance from the interface boundary to the wall corresponds to $y^{+}=100$. In general, it has been noted that the TBL model has a good enough accuracy in a quite large range of $y^{+}$, up to 300 . To make sure that this requirement is satisfied, preliminary estimates for $y^{+}$at the interface boundary might be needed.

The obtained results are compared with respect to the surface friction distribution. System (2.1) is solved using the finite-volume implicit method outlined in Section 3.

\subsection{Flow past a subsonic flat plate}

For the problem on subsonic flow past a plate, the parameters of the inflow were set as in Section 5.1. The transition point $x_{\text {trip }}=0.785$ was set according to the results of searching for LTT from Section 5.1 and the transition end $x_{2}=1.25$. The computational domain has dimensions of $1.5 \times 0.3 \times 0.03$ with number of nodes $601 \times 101 \times 4$ on each directions with a refinement to the plate (second dimension). Four solutions were obtained: a single-block solution, two two-block solutions with an ANDD and a two-block solution with ENDD. Based on these calculation results, the distributions of the friction over the plate surface are compared in Fig. 4.

The ANDD-S method demonstrates a reasonably good agreement with the single-block solution including the transition region. Some difference between the solutions is caused by the simplification of the governing equations in the near-wall region that in turn leads to accelerating convergence. The ANDD-I gives a significant mismatch because of the intermittency implemented in this model. It allows us to determine an extended transition region in accordance with the previously found positions of the transition onset and its end. This leads to a more accurate prediction of the transition region. As can be seen in the next example of a supersonic flat plate problem, at the end of the transition region, which is not taken into account in the one-block calculation, the curves for the one-block solution and ANDD-I effectively merge.

\subsection{Flow past a supersonic flat plate}

In the case of a supersonic flow past a flat plate, the parameters of the inflow were set as in Section 5.2. As was obtained in that section, the transition point is situated at 
$x_{\text {trip }}=0.13$ while $x_{2}=0.33$. Similar to the previous test case, mesh of the same size is used with another refinement to the wall to satisfy condition $y^{+} \approx 1$ at the first wall cell. Similar to the subsonic case, there solutions were obtained for the comparison with respect to the friction distribution. The obtained results are compared in Fig. 5. It turns out that NDD methods demonstrate good agreement with the single-block solution, especially the ENDD method. In this test case, it is clearly seen that in the turbulent part the friction distribution in the exact decomposition exactly coincides with the single-block solution. As can be seen from the figure, if the intermittency is taken into account, the friction reaches a fully developed turbulent regime strictly after the end of transition $x_{2}$. Thus, it is demonstrated that the ANDD-I expands the capabilities of the basic SA-la model to modeling flows with an extended transition region.

Fig. 6 shows comparison of the residual as a function of the computational cost which is estimated as follows. There are two times more cells in the outer block than those in the inner block. Then, with ENDD the computation cost of one iteration in the inner block is taken as one unit and in the outer block as two units. In turn, the single-block iteration requires three units. The ANDD requires two units per iteration because governing equations (2.1) are basically solved only in the outer block. It is to be noted that the computational cost to resolve the inner block is negligible. From the comparison it can be seen that the ANDD method converges almost three times faster than the single-block and ENDD approaches. The ENDD and single-block methods converge approximately within the same time.

\subsection{A supersonic flow over compression corner}

For the test case of a supersonic flow over a compression corner, the parameters of the inflow are set as in Section 5.2. Despite the fact that the problem is considered with the same parameters of the inflow as those at the test case for the supersonic flow past a plate, the position of the transition onset differs. This is due to the propagation of disturbances from the compression angle upstream through the subsonic part of the boundary layer. Nevertheless, as the study with the $e^{N}$-method shows, this effect insignificantly changes the position of the transition. Then, lets assume that the transition onset occurs at $x_{\text {trip }}=0.13$. The computational domain and mesh are shown in Fig. 7. Along the entire wall the mesh contains 300 cells. The characteristic cell size $\Delta x_{t}$ near the trip, which is used in the SA-la model, is equal to 0.01. In turn, the mesh along the normal to the wall direction contains 60 cells refined towards the wall. To make sure that the computational results are mesh independent, computations were also carried for the number of cells equal to 80 and 100 in the normal direction, as well as the number of cells in the longitudinal direction such that $\Delta x_{t}=0.005 ; 0.001$. As demonstrated in Fig. 8, again the NDD approaches are in a good agreement with the single-block solution. As expected, the solution obtained with ENDD is more accurate than that obtained with ANDD. 


\subsection{A planar shock wave impinging on a turbulent boundary layer}

According to $[37,38]$, the problem of a planar shock wave impinging on a turbulent boundary layer is set and solved. The transition onset is determined in [38]. A fragment of the computational domain with mesh is shown in Fig. 9. The mesh contains 346 cells along the plate. The characteristic cell size $\Delta x_{t}$ is equal to 0.0048 . In the normal direction to the wall the mesh contains 70 cells refined towards to the wall. The obtained results practically coincide the prediction reached on a finer mesh with 100 cells along the normal direction and $\Delta x_{t}=0.001$. In turn, the flow field is shown in Fig. 10.

The flat plate is situated in plane $y=0$ between $x=0$ and $x=0.32 \mathrm{~m}$. The free stream is a supersonic air flow with Mach number $M_{\infty}=3.04$ and Reynolds number $R e=38 \cdot 10^{6}$ $m^{-1}$. The transition onset occurs at $x_{\text {trip }}=0.1$. The turbulent boundary layer on the plate interacts with a planar shock wave, moved away from the cylinder of radius $r=0.01$ centered at point $(0.189,0.1)$. In Fig. 11, the friction over the plate surface obtained with ANDD-S is compared against one-block solution. As can be seen, the results occur in a reasonably good agreement.

\section{Conclusion}

The method of the near-wall decomposition has been extended to modeling non-equilibrium flows with a laminar-turbulent transition. The compressible RANS equations are closed with the use of the Spalart-Allmaras model with the trip term. The laminar-turbulent transition is taken into account in the approximate domain decomposition method by using different viscosity profiles in the laminar, transitional and turbulent regions. It has been shown that the implementation of the intermittency in the NDD approach essentially expands the capabilities of the SA-la model to efficiently model non-equilibrium turbulent flows with an extended transition region. In turn, the exact domain decomposition method can be used to model LTT straightforward similar to the fully developed turbulence modeling although it requires more computing time. The considered test cases with flows over sub- and supersonic flat plate, compression corner and a planar shock wave impinging on a turbulent boundary layer demonstrate the capacity of the NDD approach in predicting non-equilibrium flows with high accuracy. Our study shows that for the considered geometries the skin friction obtained with NDD almost coincides with the single-block solution. A slight difference is only found in the skin friction if the interface boundary is set: $y^{+}>100$. The computations for compression corner and a planar shock wave impinging on a turbulent boundary layer demonstrate the capability of the NDD method to predict with high accuracy the position of separation point and formation of the shock wave with the presence of a laminar-turbulent transition. 


\section{Acknowledgement}

The authors are grateful to V.A. Titarev for useful discussions and to the unknown referees for helpful remarks which improved the quality of the paper.

References

[1] Spalart, P. \& Allmaras, S. (1992). A one-equation turbulence model for aerodynamic flows. In 30th aerospace sciences meeting and exhibit (p. 439).

[2] Fedorov, A. \& Obraz, A. (2017). A hybrid LST-RANS method for modeling of laminarturbulent transition. 7th European conference for aeronautics and space sciences (EUCASS)

[3] Grotjans, H. \& Menter, F. R. (1998). Wall functions for industrial applications. Computational fluid dynamics, 98(1), 2.

[4] Kalitzin, G., Medic, G., Iaccarino, G. \& Durbin, P. (2005). Near-wall behavior of RANS turbulence models and implications for wall functions. Journal of Computational Physics, 204(1), 265-291.

[5] Craft, T. J., Gerasimov, A. V., Iacovides, H. \& Launder, B. E. (2002). Progress in the generalization of wall-function treatments. International Journal of Heat and Fluid Flow, $23(2), 148-160$.

[6] Craft, T. J., Gant, S. E., Iacovides, H. \& Launder, B. E. (2004). A new wall function strategy for complex turbulent flows. Numerical Heat Transfer, Part B: Fundamentals, 45(4), 301-318.

[7] Utyuzhnikov S.V. (2009). Domain decomposition for near-wall turbulent flows, Int. J. Computers \& Fluids, 38 (9): 1710-1717.

[8] Utyuzhnikov, S.V. (2014). Towards development of unsteady near-wall interface boundary conditions for turbulence modeling. Comp. Phys. Communic., 185, 11, 2879-2884.

[9] Jones, A., Utyuzhnikov, S.V. (2015). Application of a near-wall domain decomposition method to turbulent flows with heat transfer. Computers \& Fluids, 119, 87-100.

[10] Jones, A., Utyuzhnikov, S.V. (2016). A near-wall domain decomposition approach in application to turbulent flow in a diffuser. Applied Mathematical Modelling, 40, (1): 329-342.

[11] Jones, A., Utyuzhnikov, S.V. (2017). Efficient computation of turbulent flow in ribbed passages using a non-overlapping near-wall domain decomposition method, Computer Physics Communications, 217: 1-10.

[12] Utyuzhnikov, S., Petrov, M., Chikitkin, A., Titarev, V. (2018). On Extension of Near-Wall Non-overlapping Domain Decomposition to Essentially Unsteady Turbulent Flows. In International Conference on 50 years of the development of grid-characteristic method (pp. 199-209). Springer, Cham.

[13] Petrov, M., Utyuzhnikov, S., Chikitkin, A. \& Titarev, V. (2020). On extension of near-wall domain decomposition to turbulent compressible flows. Computers \& Fluids, 210, 104629.

[14] Chikitkin, A., Utyuzhnikov, S., Petrov, M. \& Titarev, V. (2020). Non-overlapping domain decomposition for modeling essentially unsteady near-wall turbulent flows. Computers \& Fluids, 104506.

[15] Utyuzhnikov, S. \& Smirnova, N. (2020). Unsteady interface boundary conditions for near-wall turbulence modeling. Computers \& Mathematics with Applications, 79(5), 1483-1502.

[16] Aleksin, V. A. \& Utyuzhnikov, S. V. (2014). Implementation of near-wall boundary conditions for modelling boundary layers with free-stream turbulence. Applied Mathematical Modelling, 38(14), 3591-3606. 
[17] Petrov, M. N., Tambova, A. A., Titarev, V. A., Utyuzhnikov, S. V. \& Chikitkin, A. V. (2018). FlowModellium software package for calculating high-speed flows of compressible fluid. Computational Mathematics and Mathematical Physics, 58(11), 1865-1886.

[18] Harten, A., Lax, P. D., \& Leer, B. V. (1983). On upstream differencing and Godunov-type schemes for hyperbolic conservation laws. SIAM review, 25(1), 35-61.

[19] Toro, E. F., Spruce, M., \& Speares, W. (1994). Restoration of the contact surface in the HLL-Riemann solver. Shock waves, 4(1), 25-34.

[20] Yoon, S. \& Jameson, A. (1988). Lower-upper symmetric-Gauss-Seidel method for the Euler and Navier-Stokes equations. AIAA journal, 26(9), 1025-1026.

[21] Menshov, I. (1995). An Implicit Advection Upwind Splitting Scheme for Hypersonic Air Flows in Thermomechanical Nonequilibrium. In 6th Int. Symp. on CFD, 1995 (Vol. 2, pp. 815-820).

[22] Petrov, M. N., Titarev, V. A., Utyuzhnikov, S. V. \& Chikitkin, A. V. (2017). A multithreaded OpenMP implementation of the LU-SGS method using the multilevel decomposition of the unstructured computational mesh. Computational Mathematics and Mathematical Physics, $57(11), 1856-1865$.

[23] Chikitkin, A., Petrov, M., Titarev, V., \& Utyuzhnikov, S. (2018). Parallel Versions of Implicit LU-SGS Method. Lobachevskii Journal of Mathematics, 39(4), 503-512.

[24] Duprat, C., Balarac, G., Metais, O., Congedo, P. M. \& Brugiere, O. (2011). A wall-layer model for large-eddy simulations of turbulent flows with/out pressure gradient. Physics of Fluids, 23(1), 015101.

[25] Allmaras, S. R. \& Forrester T. J. (2012). Modifications and clarifications for the implementation of the Spalart-Allmaras turbulence model. Seventh international conference on computational fluid dynamics (ICCFD7).

[26] Dhawan, S. J., \& Narasimha, R. (1958). Some properties of boundary layer flow during the transition from laminar to turbulent motion. Journal of Fluid Mechanics, 3(4), 418-436.

[27] Lions, P.L. (1990). On the Schwarz alternating method III: a variant for nonoverlapping subdomains, in Third International Symposium on Domain Decomposition Methods for Partial Differential Equations, T.F. Chan, R. Glowinski, J. Periaux, and O.B. Widlund, eds., SIAM, Philadelphia, PA, pp. 202-223.

[28] Deng, Q. (1997) A analysis for a nonoverlapping domain decompositon iterative procedure . SIAM J. Sci. Comp. 18: 1517-1525.

[29] Lui, S. H. (2001). On accelerated convergence of nonoverlapping Schwarz methods. Journal of computational and applied mathematics 130.1-2:309-321.

[30] Utyuzhnikov, S., Chen, W. (2019) Exact non-overlapping domain decomposition for near-wall turbulence modeling. Computers \& Fluids 181 : 283-291.

[31] Mack, L. M. (1975). A numerical method for the prediction of high-speed boundary-layer transition using linear theory. in: Aerodynamic Analyses requiring Advanced Computers NASA SP-347.

[32] Malik, M. R. (1990). Numerical methods for hypersonic boundary layer stability. J. Computational Physics, 86(2), 376-413.

[33] Khorrami, M. R., \& Malik, M. R. (1993). Efficient computation of spatial eigenvalues for hydrodynamic stability analysis. J. Computational Physics, 104(1), 267-272.

[34] Lehoucq, R. B., Sorensen, D. C. \& Yang, C. (1998). ARPACK users' guide: solution of largescale eigenvalue problems with implicitly restarted Arnoldi methods. Society for Industrial and Applied Mathematics.

[35] Mack, L. M. (1984). Special course on stability and transition of laminar flow. AGARD report, 709. 
[36] Schubauer, G. B. \& H. K. Skramstad. "Laminar-Boundary-Layer Oscillations and Transition on a Flat Plate. 1948." Report/Patent Number: NACA-TR-909.

[37] Souverein, L. J., Dupont, P., Debieve, J. F., Dussauge, J. P., Van Oudheusden, B. W. \& Scarano, F. (2010). Effect of interaction strength on unsteadiness in shock-wave-induced separations. AIAA journal, 48(7), 1480-1493.

[38] Toure, P. S. \& Schuelein, E. (2018). Numerical and experimental study of nominal 2-D ShockWave/Turbulent Boundary Layer Interactions. In 2018 Fluid Dynamics Conference (p. 3395).

[39] Utyuzhnikov S.V. (2006). The method of boundary condition transfer in application to modeling near-wall turbulent flows, Int. J. Computers \& Fluids, 35 (10): 1193-1204.

[40] Utyuzhnikov, S.V. (2012). Interface boundary conditions in near-wall turbulence modeling, Int. J. Computers \& Fluids, 68: 186-191.

[41] Utyuzhnikov, S. V. (2005). Some new approaches to building and implementation of wallfunctions for modeling of near-wall turbulent flows. Computers \& fluids, 34(7), 771-784. 
Appendix

The non-zero elements of the coefficient in (5.1) for 3D incompressible flow for matrices $A, B$ and $C$ are given below [33]. For results presented in this paper, it is set $\beta=0$ :

$$
\begin{aligned}
& A_{21}=1, A_{32}=1, B_{12}=1, B_{33}=-R, \\
& C_{11}=i \alpha, C_{21}=i \omega R-\alpha i R u_{1}, C_{22}=-R \frac{d u_{1}}{d y}, \\
& C_{23}=-i \alpha R, C_{32}=i \omega R-\alpha i R u_{1} .
\end{aligned}
$$

In the case of $3 \mathrm{D}$ compressible flow, the non-zero elements of the coefficient in (5.1) for matrices $A, B$ and $C$ are presented below [32]. In the present paper, it is set $u_{3}=0$ :

$$
\begin{aligned}
& A_{11}=1, A_{22}=1, A_{44}=1, A_{55}=1, \\
& B_{11}=\frac{1}{\mu} \frac{d \mu}{d T} T^{\prime}, B_{12}=i \alpha l_{1}, \\
& B_{14}=\frac{1}{\mu} \frac{d \mu}{d T} u_{1}^{\prime}, B_{21}=i \alpha l_{1} / l_{2}, \\
& B_{22}=\frac{1}{\mu} \frac{d \mu}{d T} T^{\prime}, B_{23}=-R /\left(l_{2} \mu\right), \\
& B_{25}=i \beta l_{1} / l_{2}, B_{32}=1, \\
& B_{41}=2(\gamma-1) \sigma M^{2} u_{1}^{\prime}, B_{44}=2 k^{\prime} / k, \\
& B_{45}=2(\gamma-1) \sigma M^{2} u_{3}^{\prime}, B_{52}=i \beta l_{1}, \\
& B_{54}=\frac{1}{\mu} \frac{d \mu}{d T} u_{3}^{\prime}, B_{55}=\frac{1}{\mu} \frac{d \mu}{d T} T^{\prime},
\end{aligned}
$$




$$
\begin{aligned}
& C_{11}=-i \xi R /(\mu T), C_{12}=-R u_{1}^{\prime} /(\mu T)+\frac{i \alpha}{\mu} \frac{d \mu}{d T} T^{\prime}, \\
& C_{13}=-i \alpha R / \mu, C_{14}=\frac{1}{\mu} \frac{d \mu}{d T} u_{1}^{\prime \prime}+\frac{1}{\mu} \frac{d^{2} \mu}{d T^{2}} T^{\prime} u_{1}^{\prime}, \\
& C_{15}=-\alpha \beta l_{1}, C_{21}=i \alpha \frac{1}{\mu} \frac{d \mu}{d T} T^{\prime} l_{0} / l_{2} \\
& C_{22}=-i \xi R /\left(l_{2} \mu T\right), C_{24}=i \frac{1}{\mu} \frac{d \mu}{d T} T^{\prime}\left(\alpha u_{1}^{\prime}+\beta u_{3}^{\prime}\right) \\
& C_{25}=i \beta \frac{1}{\mu} \frac{d \mu}{d T} T^{\prime} l_{0} / l_{2}, C_{31}=i \alpha, C_{32}=-T^{\prime} / T, \\
& C_{33}=i \gamma M^{2} \xi, C_{34}=-i \xi / T, C_{35}=i \beta, \\
& C_{51}=-\alpha \beta l_{1}, C_{52}=i \beta \frac{1}{\mu} \frac{d \mu}{d T} T^{\prime}-R u_{3}^{\prime} /(\mu T), \\
& C_{53}=-i \beta R / \mu, C_{54}=\frac{1}{\mu} \frac{d \mu}{d T} u_{3}^{\prime \prime}+\frac{1}{\mu} \frac{d^{2} \mu}{d T^{2}} T^{\prime} u_{3}^{\prime}, \\
& C_{55}=-i \xi R /(\mu T), \\
& C_{42}=2 i(\gamma-1) M^{2} \sigma\left(\alpha u_{1}^{\prime}+\beta u_{3}^{\prime}\right)-\sigma R T^{\prime} /(\mu T), \\
& C_{43}=i \xi(\gamma-1) M^{2} \sigma R / \mu, \\
& C_{44}=-i \xi R \sigma /(\mu T)+(y-1) M^{2} \sigma \frac{1}{\mu} \frac{d \mu}{d T}\left(u_{1}^{\prime 2}+u_{3}^{\prime 2}\right)+k^{\prime \prime} / k .
\end{aligned}
$$




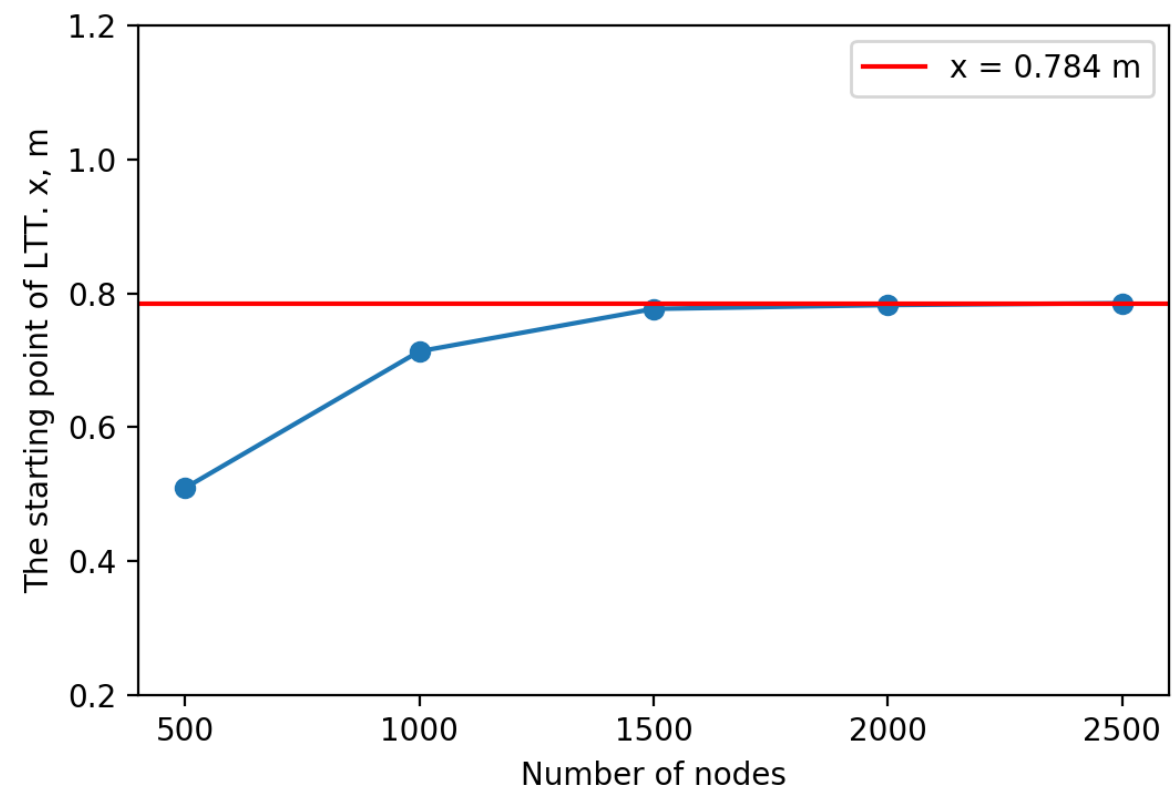

Figure 1: Dependence of transition onset on the number of nodes for subsonic flow past a flat plate. 


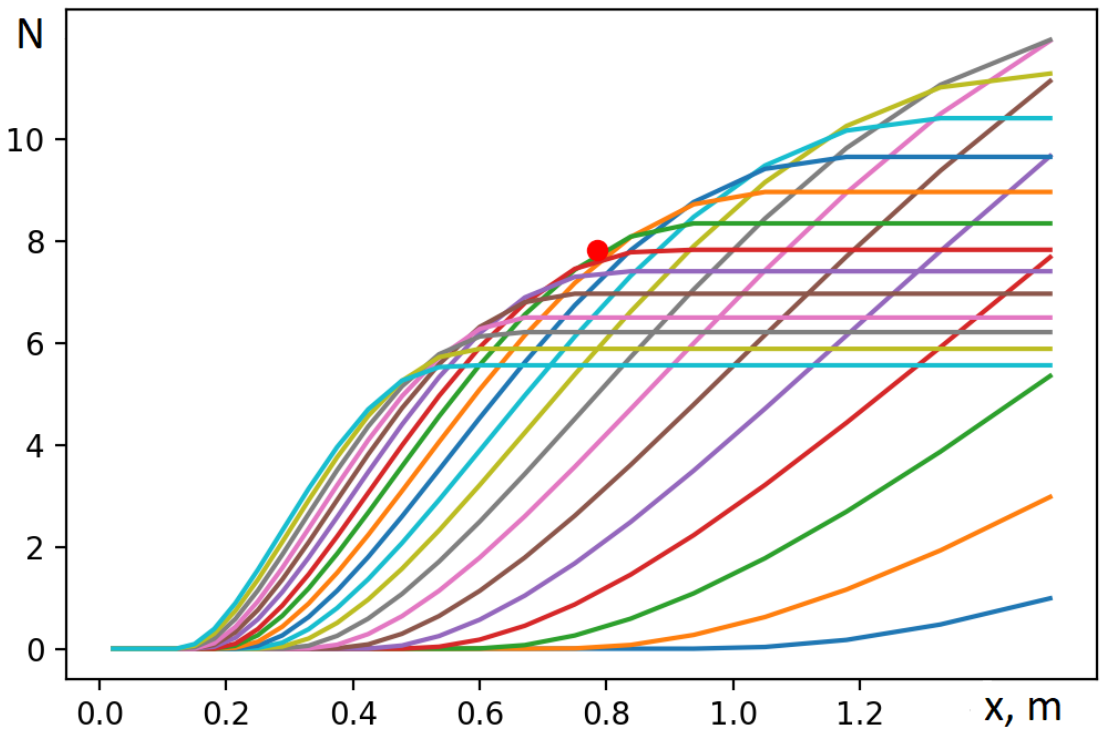

Figure 2: Amplification curves for search for transition in a subsonic flow past a flat plate. The red dot marks the position on the envelope corresponding to the beginning of LTT. 


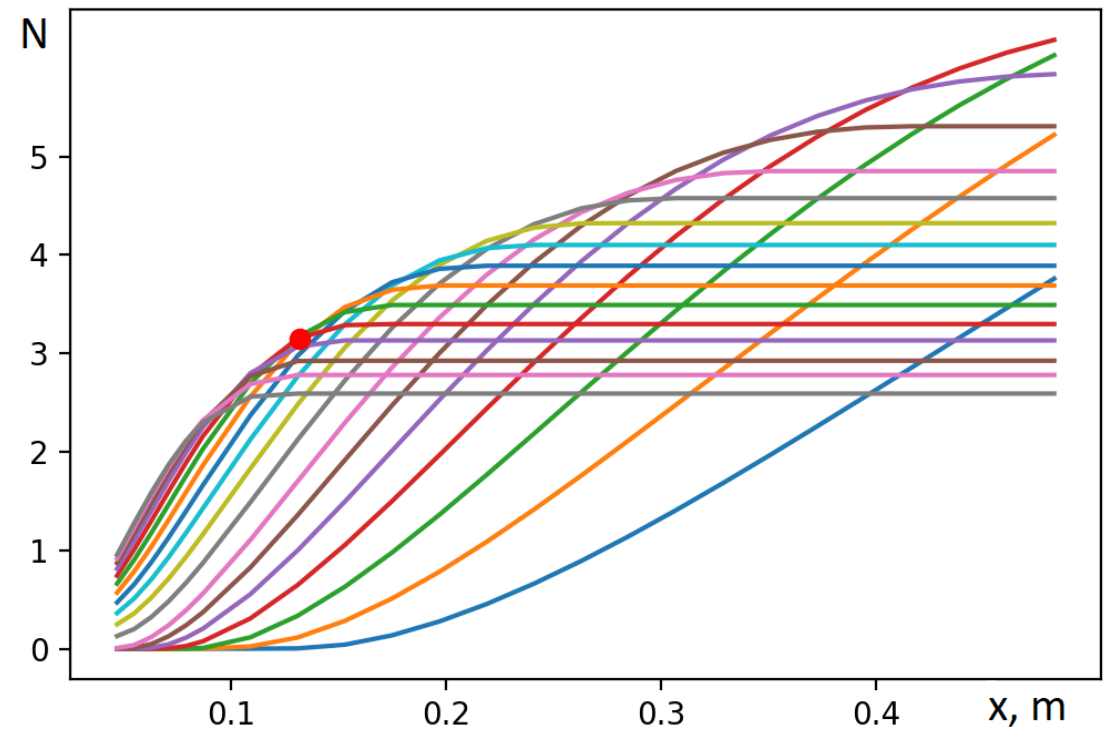

Figure 3: Amplification curves for search for transition in a supersonic flow past a flat plate. The red dot marks the position on the envelope corresponding to the beginning of LTT. 


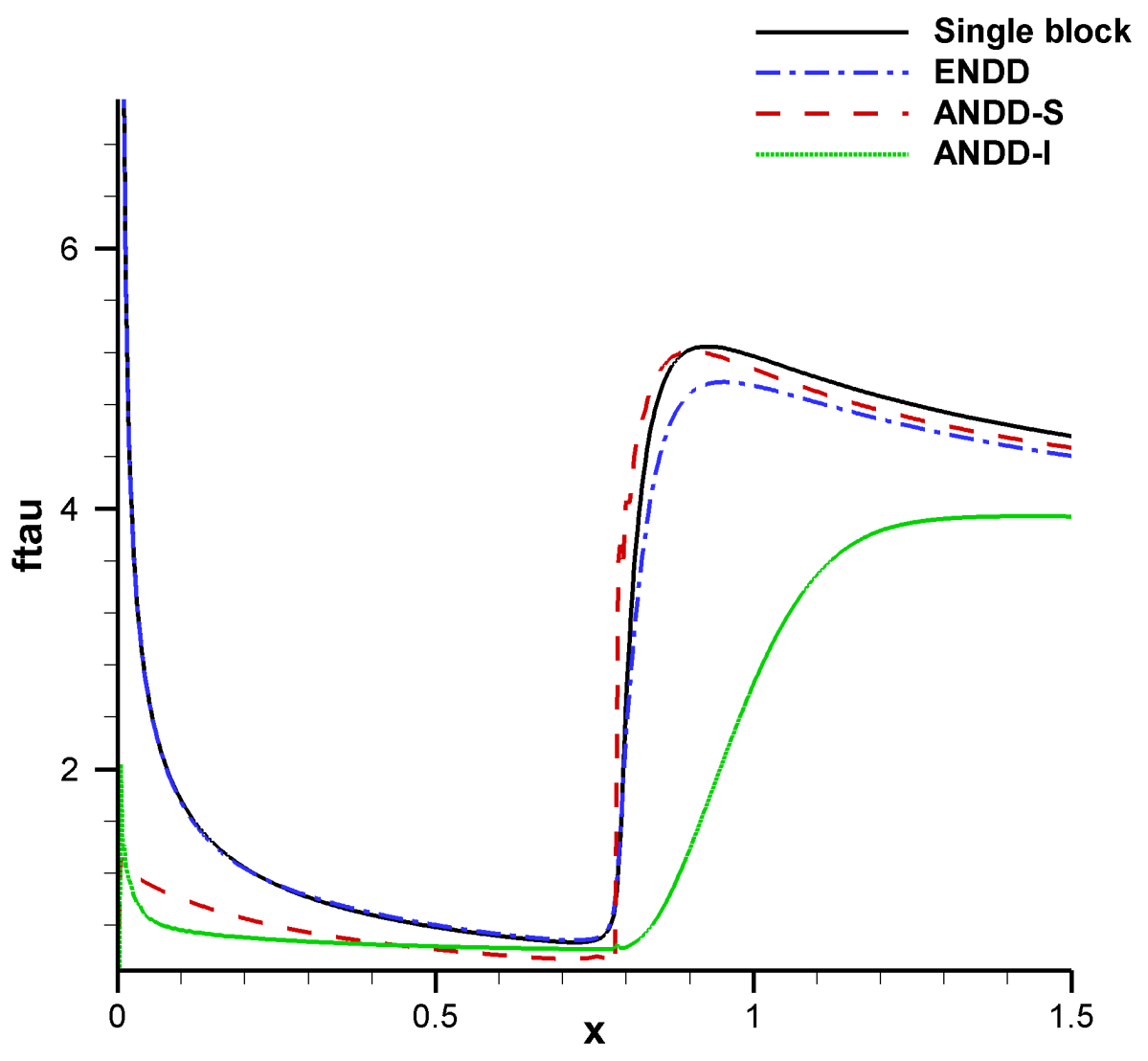

Figure 4: Comparison of friction in a single-block calculation (single block) against twoblock solutions with ENDD, ANDD-S and ANDD-I for subsonic flow past a flat plate. 


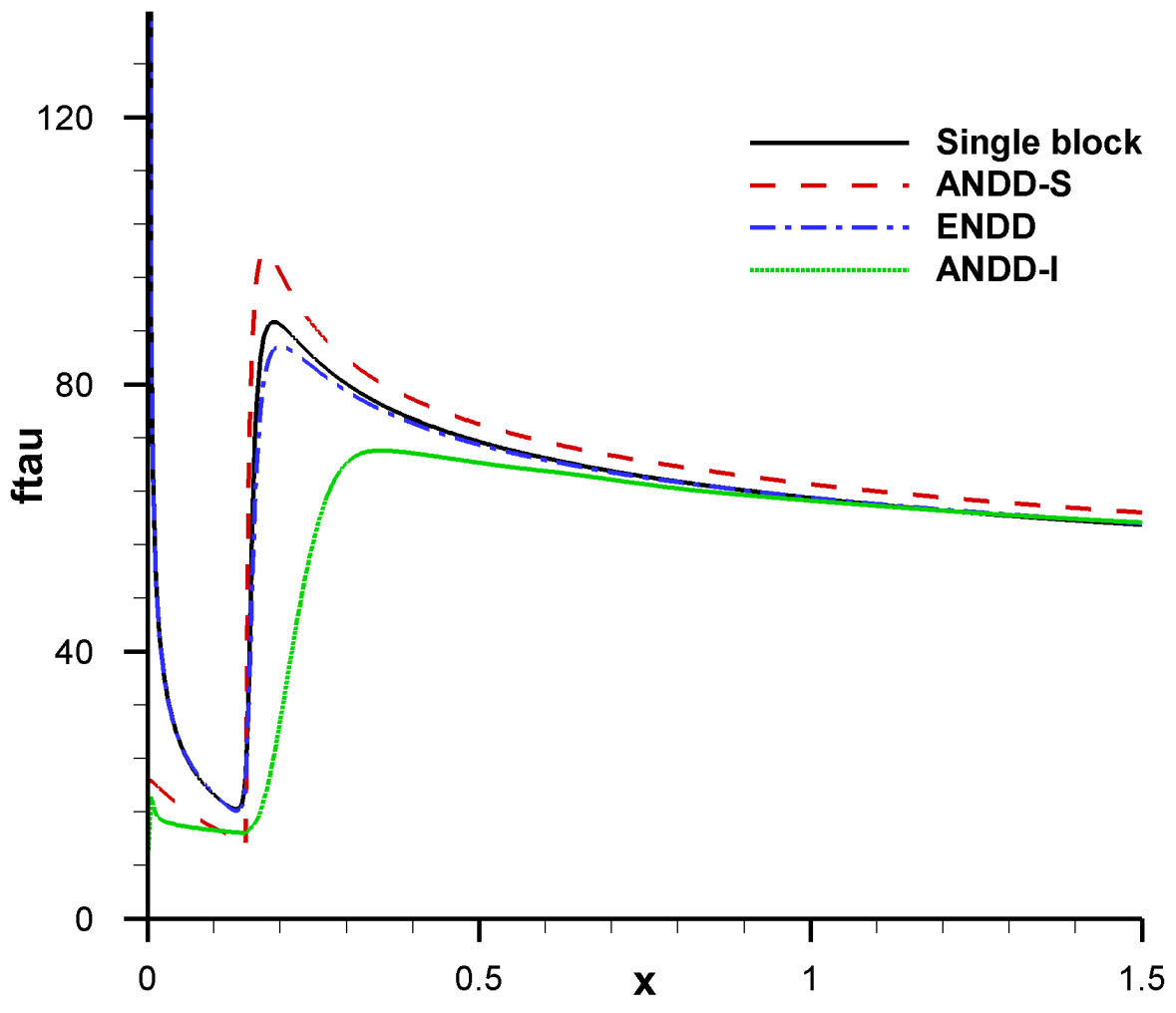

Figure 5: Comparison of friction in a single-block calculation (single block) against twoblock solutions with ENDD, ANDD-S and ANDD-I for supersonic flow past a flat plate. 


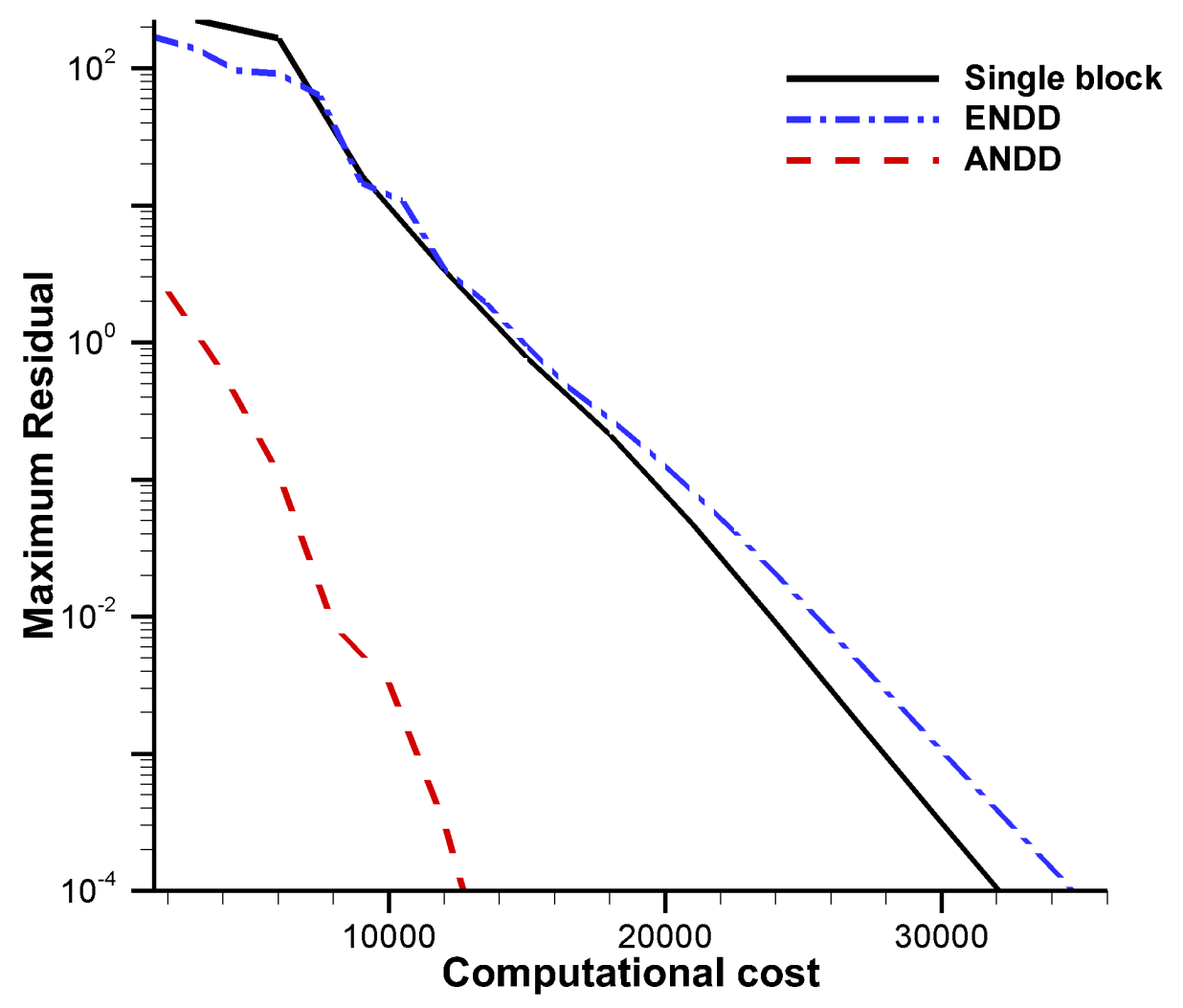

Figure 6: Comparison of the residual time history for ANDD, ENDD and single-block computations for a supersonic flow past a flat plate with LTT. The computational cost is measured in conventional units. 


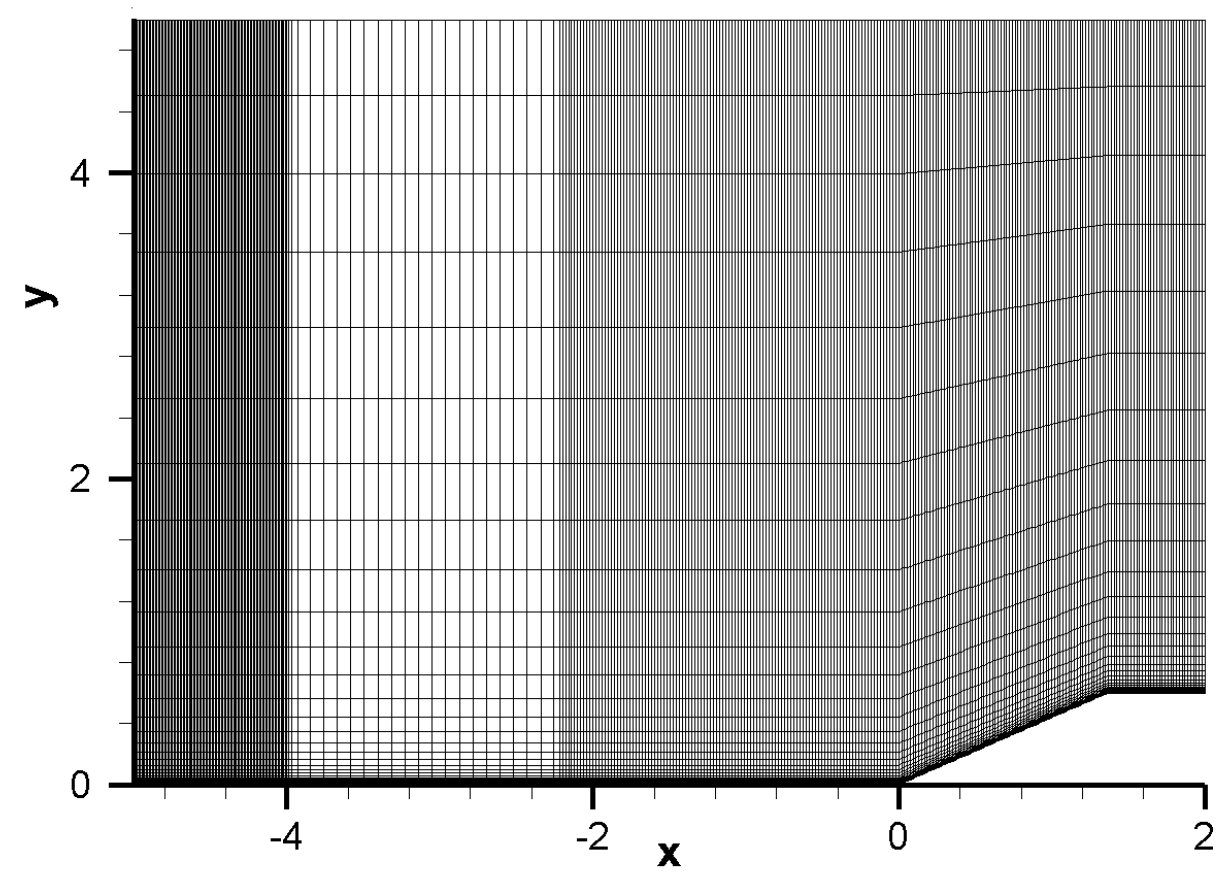

Figure 7: A supersonic flow over compression corner mesh. 


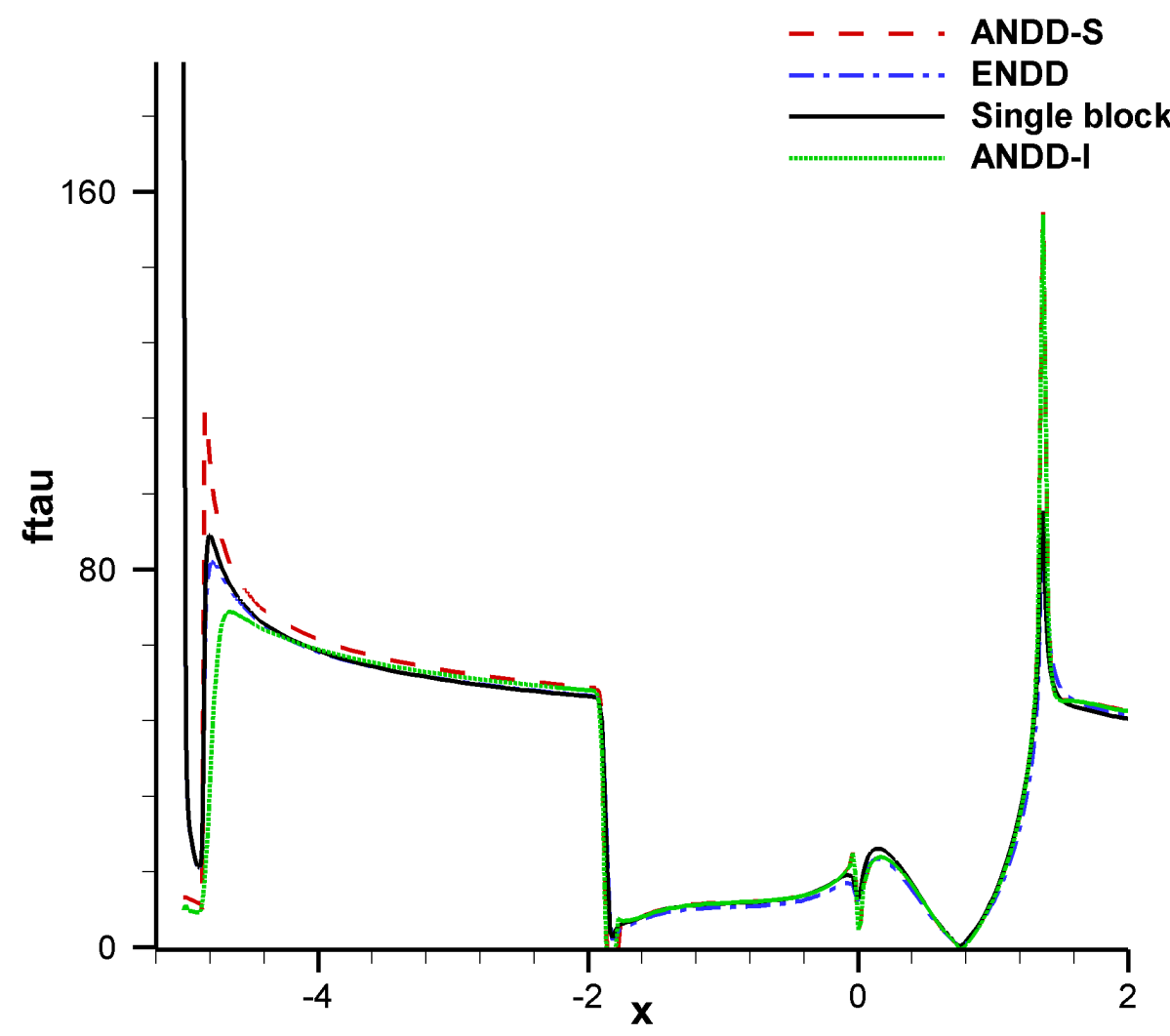

Figure 8: Supersonic flow over compression corner. Comparison of friction in a single block solution against with two-block solutions with ENDD, ANDD-S and ANDD-I. 


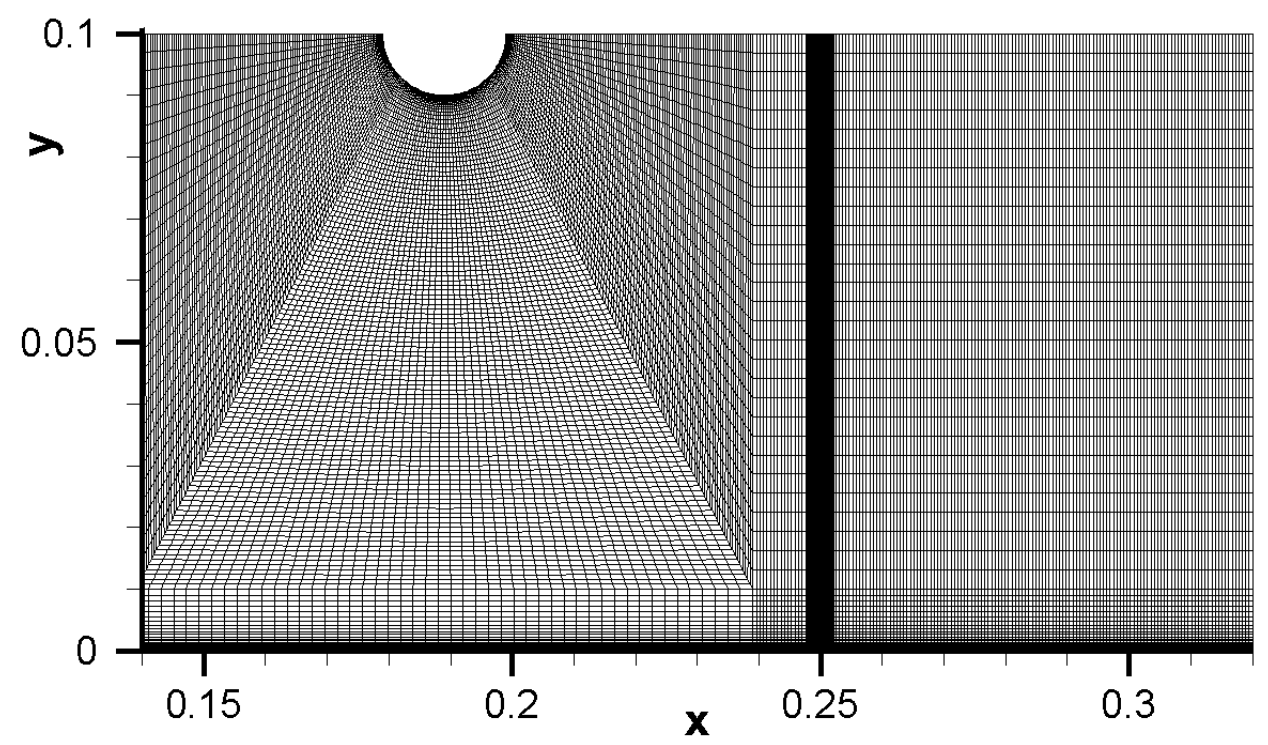

Figure 9: Fragment of the computational domain with mesh for a planar shock wave impinging on a turbulent boundary layer.

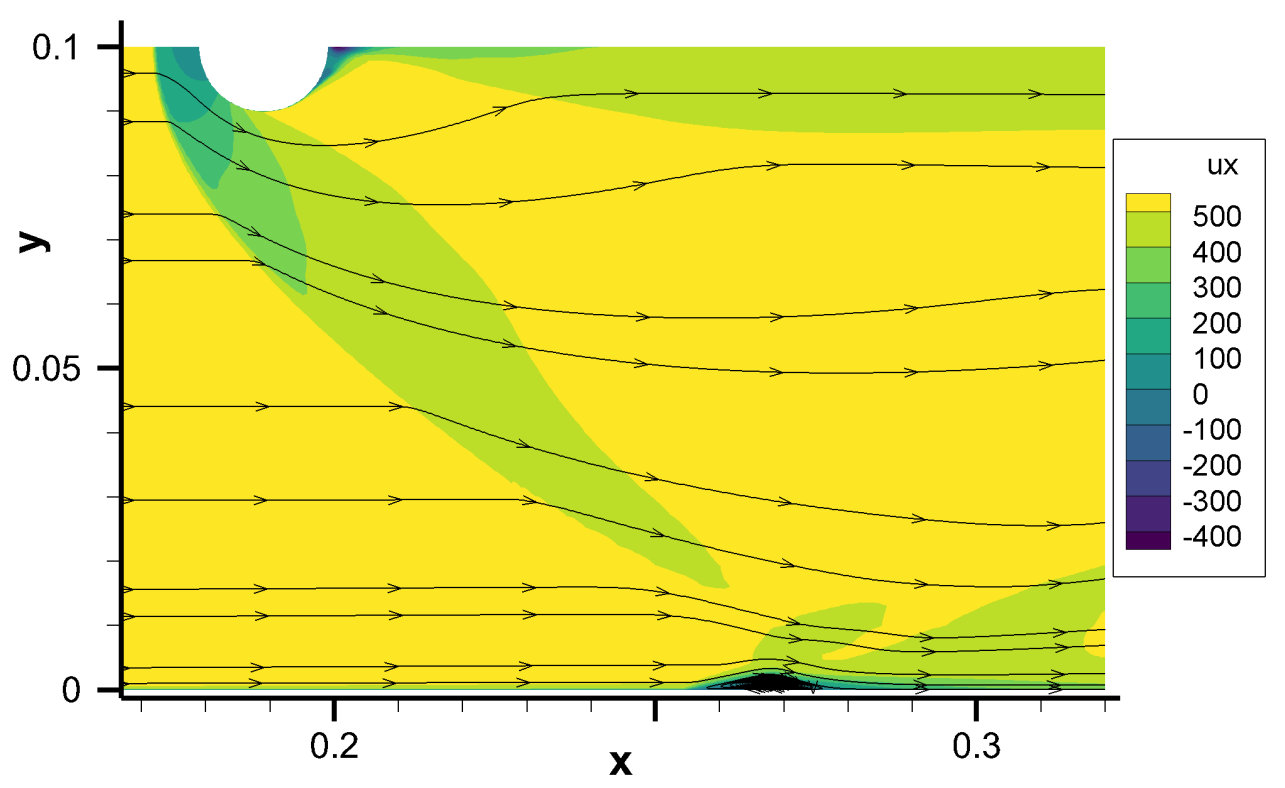

Figure 10: Flow field in the problem of a planar shock wave impinging on a turbulent boundary layer. 


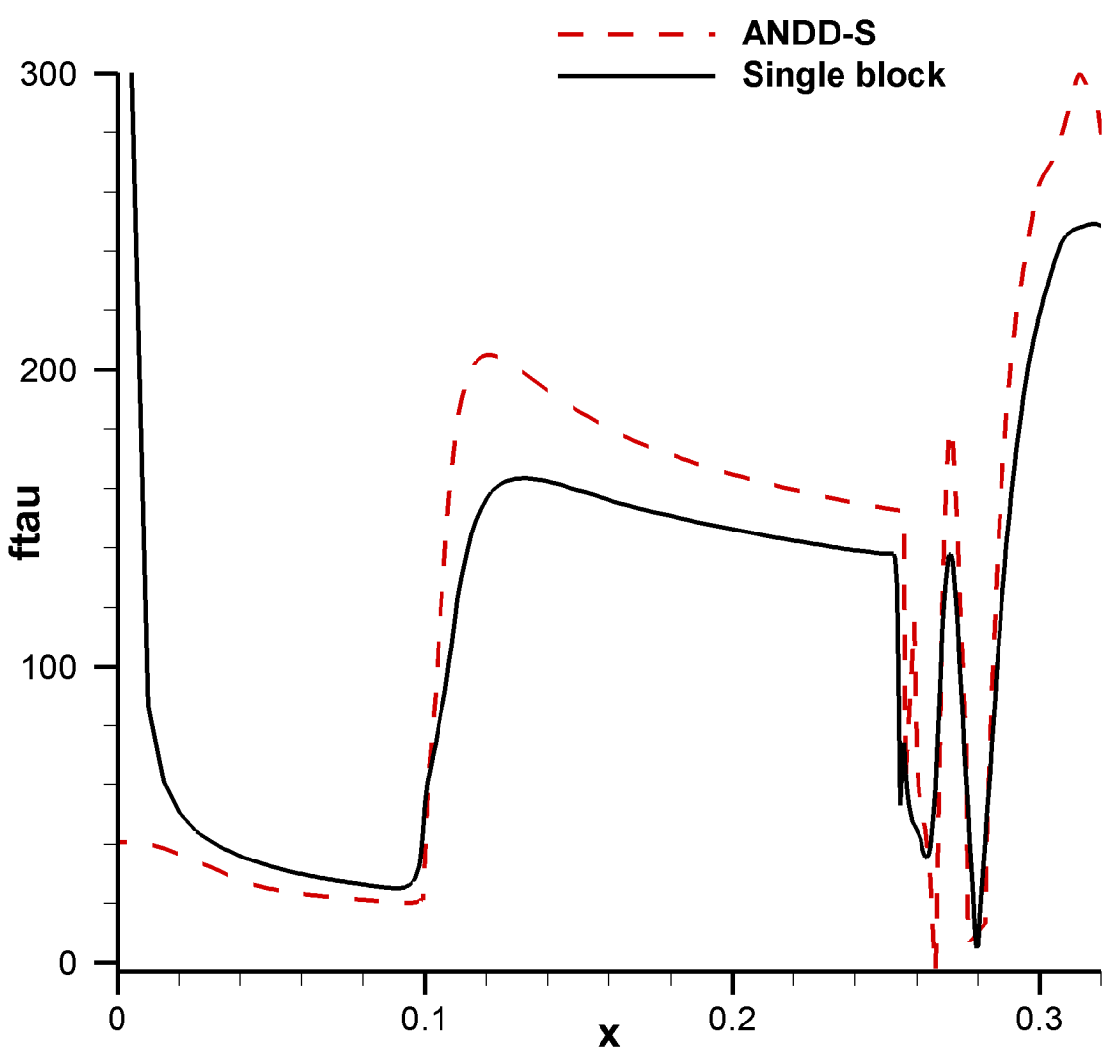

Figure 11: Planar shock wave impinging on a turbulent boundary layer. Comparison of friction for a single-block solution against a two-block solution with ANDD-S. 